
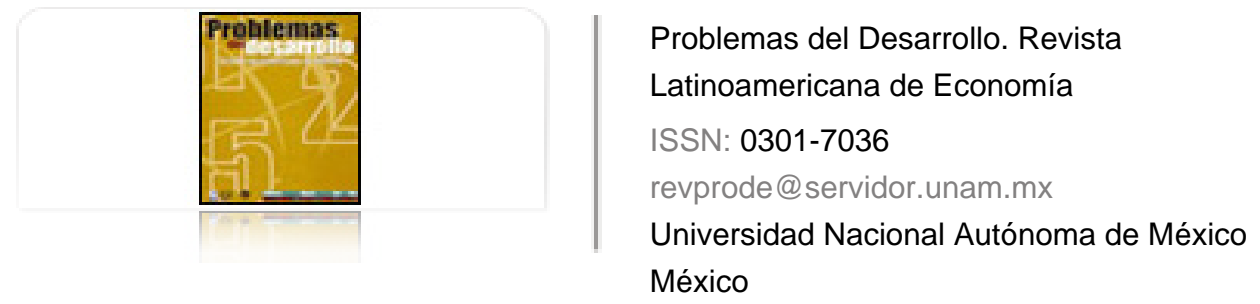

Hernández, Luis Enrique

La renta petrolera y su impacto en el crecimiento económico de Venezuela

Problemas del Desarrollo. Revista Latinoamericana de Economía, vol. 37, núm. 145, abril-junio, 2006,

pp. 135-151

Universidad Nacional Autónoma de México

Distrito Federal, México

Disponible en: http://www.redalyc.org/articulo.oa?id=11820086007

Cómo citar el artículo

- Número completo

- Más información del artículo

Página de la revista en redalyc.org

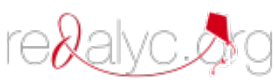

Sistema de Información Científica

Red de Revistas Científicas de América Latina, el Caribe, España y Portugal Proyecto académico sin fines de lucro, desarrollado bajo la iniciativa de acceso abierto 


\section{LA RENTA PETROLERA Y SU IMPACTO EN EL CRECIMIENTO ECONÓMICO DE VENEZUELA}

\section{Luis Enrique Hernández*}

Fecha de recepción: 26 de septiembre de 2005. Fecha de aceptación: 9 de marzo de 2006.

\section{Resumen}

En la presente investigación se lleva cabo una explicación del crecimiento de la economía venezolana en su condición rentista. A partir de la teoría de Solow se introduce la renta petrolera como variable determinante junto a otras, como las tasas de crecimiento poblacional y la de inversión; igualmente se estima la productividad total de los factores a partir de un modelo que incluye la renta. La conclusión es que si bien ésta sigue teniendo una significativa importancia en el crecimiento, la misma ha disminuido su impacto a partir de finales de los años setenta. Parece haber un debilitamiento de un modelo basado en una renta creciente, que en su momento contribuyó a expandir el crecimiento de la economía, pero que en la actualidad no ha permitido salir de la recesión económica que ya tiene más de dos décadas.

Palabras claves: renta, crecimiento, productividad de los factores, inversión

\section{Abstract}

This study seeks to explain the growth of the Venezuelan economy as a rent-earner. Based on Solow's theory, rent from petroleum is introduced as the determinant variable -in the Venezuelan case - together with other variables, such as demographic growth and investment. Likewise, total factor productivity is estimated, based on a model that includes rent. The conclusion is that while this continues to have great significance in growth, its impact has declined since the end of the 1970s. There appears to be a weakening of a model based on growing rent, which in its time helped to expand the economy's growth, but which today has not enabled Venezuela to escape from the economic recession, which has lasted now for two decades.

Key words: rent, growth, factor productivity, investment.

* Economista, investigador independiente, colaborador del Instituto de Investigaciones Económicas de la Universidad del Zulia. Correo electrónico: 1uisenriquehg@gmail.com, lehernandez@bod.com.ve 


\section{LUIS ENRIQUE HERNÁNDEZ}

\section{Résumé}

Dans cette investigation on met en place une explication de la croissance de l'économie vénézuélienne dans sa condition rentière. A partir de la théorie de Solow, on introduit la rente pétrolière comme une variable déterminante -dans le cas vénézuélien-à côté d'autres, telles que les taux de croissance de la population et de l'investissement; on prend en compte également la productivité totale des facteurs à partir d'un modèle qui inclut la rente. La conclusion est que, même si elle a toujours une importance significative dans la croissance, elle même a diminué son impact à partir de la fin des années soixante-dix. Il semble y avoir un affaiblissement d'un modèle basé sur une rente croissante, qui a contribué à un moment donné à l'expansion de la croissance de l'économie, mais qui actuellement n'a pas permis de sortir de la récession économique qui dure depuis plus de deux décades.

Mots-cléfs: rente, croissance, productivité des facteurs, investissement.

\section{Resumo}

Na presente pesquisa fornece-se uma explanação do crescimento da economia venezolana na sua condição rentista. A partir da teoria de Solow se introduz a renda petroleira como variável determinante - no caso venezolano - junto a outras tais como as taxas de crescimento populacional e a do investimento; igualmente calcula-se a produtividade total dos fatores a partir dum padrão que inclui à renda. A conclusão é que se é verdade que ela continua tendo uma grande importância no crescimento, também tem diminuído seu impacto a partir de finais dos anos 70. Percebe-se um enfraquecimento dum padrão baseado numa renda crescente, que no seu momento contribuiu à expansão do crescimento da economia, porém no presente ainda não permite sair da recessão econômica que já tem mais de duas décadas.

Palavras chave: renda, crescimento, produtividade dos fatores, investimento. 


\section{Introducción}

$\mathrm{E}$

1 tema del crecimiento es tal vez el que ha sido más estudiado a lo largo de la

historia económica. Las causas que lo propician han sido ampliamente discutidas

tanto en forma individual como de manera conjunta. Dentro de las razones causales se destacan siempre la acumulación de capital, la mejora en el capital humano, el progreso tecnológico y el comercio exterior. También ha sido objeto de debate e interpretaciones de las diferentes escuelas de pensamiento económico, resultado directo de una época, de una necesidad de respuestas a los fenómenos socioeconómicos que subyacen en las oscilaciones cíclicas, así como de las inquietudes intelectuales de cada momento.

La preocupación por el crecimiento condujo a la escuela clásica al estudio de los mercados y del sistema de precios como una forma de asignación de recursos. Aunque en principio se centró más en el tema del desarrollo, y consideraba factores culturales, políticos, sociales e históricos, su interés por la libertad de mercado, por la libre competencia y por la asignación eficiente de los recursos motivó un interés mayor por el estudio de los factores económicos impulsores del crecimiento.

Para Keynes, el crecimiento se centra necesariamente en la intervención del Estado como una solución para salir de la depresión originada por la insuficiencia en la demanda agregada. De acuerdo a su teoría, una primera medida para detener la caída de la producción y buscar relanzar el crecimiento es mediante la implantación de políticas anticíclicas, basadas en la determinación de niveles satisfactorios de demanda agregada básicamente mediante mayor gasto público.

En su análisis del crecimiento, Keynes destaca como objetivo fundamental de política económica la consecución de un determinado nivel de empleo. En este esquema, es la población la que juega un papel fundamental, pues es la que anima, con su consumo, a los empresarios a seguir desarrollando y expandiendo su actividad (Galindo y Malgasini 1994:9).

En este mismo orden de ideas, Harrod (1979:22), de la escuela neokeynesiana, expresa que "existe un nivel de producto que crece a una tasa de crecimiento natural. Y para que exista un crecimiento sostenido y equilibrado con pleno empleo, esta tasa tiene que ser igual a la tasa de crecimiento efectiva y a la de crecimiento garantizada". Ello no implica que estas tasas deban ser iguales. Harrod afirma que existe una serie de dificultades que impiden alcanzar un equilibrio con pleno empleo; no hay ningún mecanismo seguro que permita que la economía iguale su tasa natural con la garantizada.

Otra de las contribuciones importantes dentro de la noción del crecimiento, incluso previo a los aportes keynesianos, es la que desarrolló Kalecki (1977:498), quien afirmó

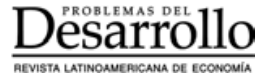


"que el rasgo principal del crecimiento es la inversión, que no va a depender del proceso multiplicador, sino que, por el contrario, va estar relacionada con el ciclo económico, que se ve afectado por las modificaciones en el proceso inversor, y éste a su vez habrá que considerarlo dentro del contexto de un país que está creciendo".

Otros autores como Gimeno, González y Ruiz (2000:365) definen el crecimiento como el aumento de la capacidad productiva en el largo plazo y ésta, a su vez, depende de los actores y de su productividad; en tal sentido, sostienen que el crecimiento se verá condicionado por factores demográficos y del mercado de trabajo, la calidad del capital humano, el capital físico disponible y la innovación tecnológica.

En el caso de una economía rentista, como la venezolana, el tema del crecimiento adquiere matices diferentes, dada la característica particular que la renta le añade al análisis. Este es precisamente el objetivo del presente trabajo: intentar comprender de qué forma la renta petrolera ha contribuido al crecimiento de dicha economía y qué consecuencias ha tenido la caída de la renta en ese proceso.

Antes de entrar en consideraciones acerca de la crisis venezolana, debemos señalar que lo que nos ocupa en este caso es la renta petrolera entendida como una renta internacional del suelo y por tanto un ingreso que no es producto del esfuerzo productivo interno. Según Baptista (1997:8), ésta es "la remuneración pagada con cargo a la propiedad sobre unos medios de producción no producidos". Para este país sudamericano la renta petrolera no es la resultante del esfuerzo productivo de los factores que participan en cualquier sociedad capitalista para generar el producto; el petróleo está ahí, nadie lo produjo.

Junto al agregado de cosas que se producen y distribuyen por el concurso del esfuerzo productivo nacional, la sociedad dispone de manera continua de un ingreso de origen internacional que es significativo respecto de la magnitud del ingreso creado internamente. Este último ingreso, que no tiene como contrapartida ni el trabajo ni el capital doméstico, sino sólo la propiedad sobre un medio de producción no producido, es también objeto de distribución entre los diferentes ingresos particulares percibidos de manera normal en la sociedad capitalista (Baptista, 1997:13).

Algunos califican la crisis venezolana como un caso de "enfermedad holandesa", en la que un boom de recursos naturales termina propiciando una apreciación real de la moneda con la consiguiente caída de la competitividad de los sectores transables de la economía. Autores como Corden (1983) han abordado este problema para países primarioexportadores, sobre todo, países petroleros. Por otro lado, Auty (1990) plantea la llamada "maldición de los recursos naturales", en la cual establece un vínculo negativo entre el sector primario exportador y el proceso industrializador de los países en desarrollo, relación que según el autor opera por medio de una sobrevaluación estructural de la moneda.

López (2001), apoyada en la tesis desarrollada previamente por autores como Baptista y Mommer (1995), plantea que más que una expresión de la llamada "enfermedad holandesa" 
o "maldición de los recursos naturales", la crisis económica que comienza a experimentar Venezuela se explica fundamentalmente porque la renta petrolera desbordó la capacidad de absorción de capital de la economía. Es decir, a causa de la fuerte política de inversión de capital como parte del proceso de industrialización por sustitución se terminó ampliando la capacidad de la economía más allá del mercado. Si bien la sobrevaluación del bolívar contribuyó al desarrollo económico del país desde los años treinta hasta principios de los setenta, al garantizar la importación barata de bienes tanto de capital como de consumo, se constituyó en un obstáculo al desarrollo una vez que el reducido tamaño del mercado estableció limites al crecimiento industrial junto al relativamente inexistente sector exportador no petrolero.

Por otro lado, Sachs y Warner (1995) plantean que los países con gran dependencia de las exportaciones de recursos naturales tienen menores tasas de crecimiento que las economías más desarrolladas. Baptista (2004) no está de acuerdo con esos planteamientos, ya que la tesis no permite explicar casos de naciones con abundantes recursos naturales que pertenecen a las economías desarrolladas, o que han logrado crecer de manera sostenida durante largos periodos como Chile y Noruega, entre otros. Según el autor el problema no radica en tener abundantes recursos naturales ni siquiera el petróleo en específico, sino en la presencia de un Estado rico y autónomo que no debe esa riqueza y autonomía a las fuerzas productivas de la economía. Esto deviene en un desequilibrio en el sistema entre el poder político (que debe radicar en el Estado) y el poder económico (que debe originarse en los sectores productivos privados).

En la presente investigación se lleva a cabo un análisis de la importancia que ha tenido la renta petrolera durante el periodo de alto crecimiento de la economía venezolana como principal fuente de financiamiento de la inversión de capital y el gasto del estado, así como durante el periodo de recesión que ha atravesado desde finales de los años setenta, vinculado a la caída de dicha renta y al agotamiento del modelo rentístico de la economía, según varios autores.

En este sentido, en la primera parte del presente trabajo se revisarán los planteamientos desarrollados por Robert Solow como principal referencia teórica para explicar el crecimiento, al igual que el cálculo del conocido residuo de este autor. Este último permitirá determinar aquellas variaciones del producto que no están asociadas a la acumulación de factores productivos o a variaciones en su intensidad de uso, sino que se asocian, más bien, al cambio tecnológico u organizacional, así como a la eficiencia en el uso de los factores productivos. Se hará uso también de los trabajos desarrollados en relación a la importancia en el caso venezolano de los recursos naturales y de la renta como elementos dinamizadores de la economía.

Es preciso acotar que si bien el modelo de Solow ha sido ampliamente usado como referencia para explicar el fenómeno del crecimiento, también presenta sus limitaciones

\section{DeSarrollo}


dado el alto nivel de agregación en su estructura, lo que deja fuera una serie de factores de importancia para el análisis. La estimación de la productividad total de los factores o residuo de Solow intenta resolver este problema recogiendo el efecto de aspectos no observados de forma directa en las variables por considerar en el modelo.

En la segunda parte se llevará a cabo la formulación de algunos modelos con la intención de aportar evidencia empírica a favor de los planteamientos desarrollados por algunos autores de cómo el efecto de la renta en la actividad económica sobre el crecimiento ha sido cada vez menor tanto de manera directa como de forma indirecta — por medio de una disminución en la inversión de capital por trabajador y su efecto adverso en el producto de la economía-. Por último, se extraerán las principales conclusiones de la presente investigación.

\section{Modelo de crecimiento de Solow}

El modelo propuesto por Solow parte de una función de producción neoclásica, en la cual el producto es una función del capital, del trabajo y de un factor asociado al cambio tecnológico de la forma: ${ }^{1}$

$$
Y=A f(K, L)
$$

Esta función puede ser expresada en términos per capita, para lo cual se parte del supuesto de que la población crece a la misma tasa que el factor trabajo. Así, el producto per capita es igual al producto por unidad de trabajo y se designa al capital per capita como capital por unidad de trabajo, quedando:

$$
y=A f(k)
$$

El producto es una función creciente de la razón capital-trabajo, pero a una tasa decreciente, dado que la productividad marginal de los factores capital y trabajo lo son.

Partiendo del supuesto de una economía cerrada y en ausencia de cambio tecnológico, se tiene que la inversión es igual al ahorro $(I=S)$, de esta forma, desaparece el problema de un posible exceso o insuficiencia de demanda que juega un papel fundamental en la teoría keynesiana. Por otro lado, la depreciación es una proporción fija del capital ' $K$. Igualmente, se supone que el ahorro es una proporción del producto $s Y$. De esta forma, un cambio en el stock de capital viene dado por:

1 Los cambios en el estado de la tecnología causarán incrementos iguales en la productividad marginal de los factores capital y trabajo.

\section{DeSarrollo}




$$
\Delta K=s Y-\delta K
$$

Si se expresa esta función en términos per capita queda que:

$$
\frac{\Delta K}{L}=s y-\delta k
$$

Ahora bien, como la población crece a la misma tasa de la fuerza de trabajo, la cual crece a una tasa $n$, entonces una fracción del ahorro per capita debe ser usada para equipar a los nuevos trabajadores que entran a la economía. Para alcanzar este propósito se debe aplicar un monto $n k$ del ahorro. En este esquema, la variación en el capital por trabajador queda representada de la siguiente forma:

$$
\Delta k=s y-(n+\delta) k
$$

El modelo predice un equilibrio de largo plazo, conocido como estado estacionario, ${ }^{2}$ en el cual $k^{*}=y^{*}=0$. Para $\Delta k=0$ se debe cumplir que:

$$
s y=(n+\delta) k
$$

En el estado estacionario la tasa de ahorro es exactamente igual a la caída del stock de capital per capita asociado a la depreciación y al crecimiento de la población, manteniendo constante tanto al capital por trabajador como el producto per capita. ${ }^{3}$

Así, en forma resumida, el modelo de Solow establece que el capital está relacionado en forma positiva con el ahorro y negativa con el aumento de la población. El modelo parte de los supuestos de que existen rendimientos constantes a escala y competencia perfecta que son la base en la formalización del conocido equilibrio general de Walras; es decir, permite la posibilidad de sustituir o realizar diferentes combinaciones de capital y trabajo para obtener una determinada cantidad de producción.

Ahora bien, ¿qué papel juega la inclusión de la renta petrolera dentro de este modelo? Para ello, veamos el trabajo desarrollado por Schlisser y Silva (2000) acerca de la economía venezolana. Primero, si se parte de la función de producción presentada en (1) y se incluye

El estado estacionario se refiere a la situación en la cual las variables crecen a una tasa constante. Aun cuando en el estado estacionario tanto $y$ como $k$ se mantienen constantes no significa que el crecimiento sea cero; de hecho, el producto agregado crece a una tasa igual a $n$. Si la fuerza de trabajo está creciendo a dicha tasa, entonces, el capital está creciendo a la misma tasa, dado que la relación capital trabajo es constante. Esto es, $\Delta K / K=\Delta L / L=n$. Por lo tanto, como $L$ y $K$ crecen a la tasa $n$, el producto crecerá a la tasa $n$, dado que el producto per capita es constante; esto es, $\Delta Y / Y=$ $\Delta L / L=n$

\section{DeSarrollo}


la renta como otro factor determinante del crecimiento, que a su vez no depende del grado de utilización de ninguno de los otros dos factores, queda:

$$
Y=A f(K, L)+R
$$

En términos per capita se tiene que:

$$
y=a f(k)+r
$$

Dado que una importante porción de la renta en el caso venezolano ha sido destinada a expandir la economía interna por medio de programas masivos de inversión acometidos por el Estado, ${ }^{4}$ el cual se apropia y distribuye la renta petrolera, la función de acumulación de capital expresado en (5) sufriría una modificación al incorporar como factor determinante dicha renta; expresado en términos per capita, queda:

$$
\Delta k=\beta r+s y-(n+\delta) k
$$

La inclusión de la renta petrolera tiene una significativa importancia, de acuerdo con los autores, dado que en el supuesto del estado estacionario en donde $k=y=0$, un incremento en la renta petrolera con un $\beta>0$ producirá un incremento en la acumulación de capital, y por ende, mayor crecimiento económico. El stock de capital crecerá trayendo consigo mayor depreciación, lo cual ameritará tasas crecientes de inversión para poder mantener las tasas de crecimiento. En este escenario, cuando la renta petrolera comience a descender, la inversión, dado el stock de capital existente, no será suficiente para reponer los bienes de capital depreciado y se generará un proceso de desacumulación de capital con la consiguiente caída en el crecimiento de la economía.

Rodríguez y Sach (1999) plantean que el colapso de la economía venezolana tiene su origen en el proceso de sobreinversión que se experimentó durante el boom petrolero de los setenta, que acercó a la economía a un equilibrio de largo plazo desde un nivel superior. Según los autores, el proceso de acumulación se revierte dado que la demanda no responde a la misma velocidad que el producto, con lo que se genera un sobrecalentamiento de la economía. Si a esto se le suma una caída en la renta, la desacumulación de capital se acentúa y el proceso de crecimiento se revierte. En la Gráfica 1 se puede apreciar cómo la inversión comienza a caer de forma ininterrumpida poco después de que la renta lo hace. A partir de finales de los setenta la relación es casi perfecta entre ambas variables.

4 Los esfuerzos del Estado estaban orientados a las políticas de industrialización por sustitución de importaciones y al proceso de urbanización, lo cual requería una ingente cantidad de recursos orientados a la inversión en capital físico.

\section{DeSarrollo}



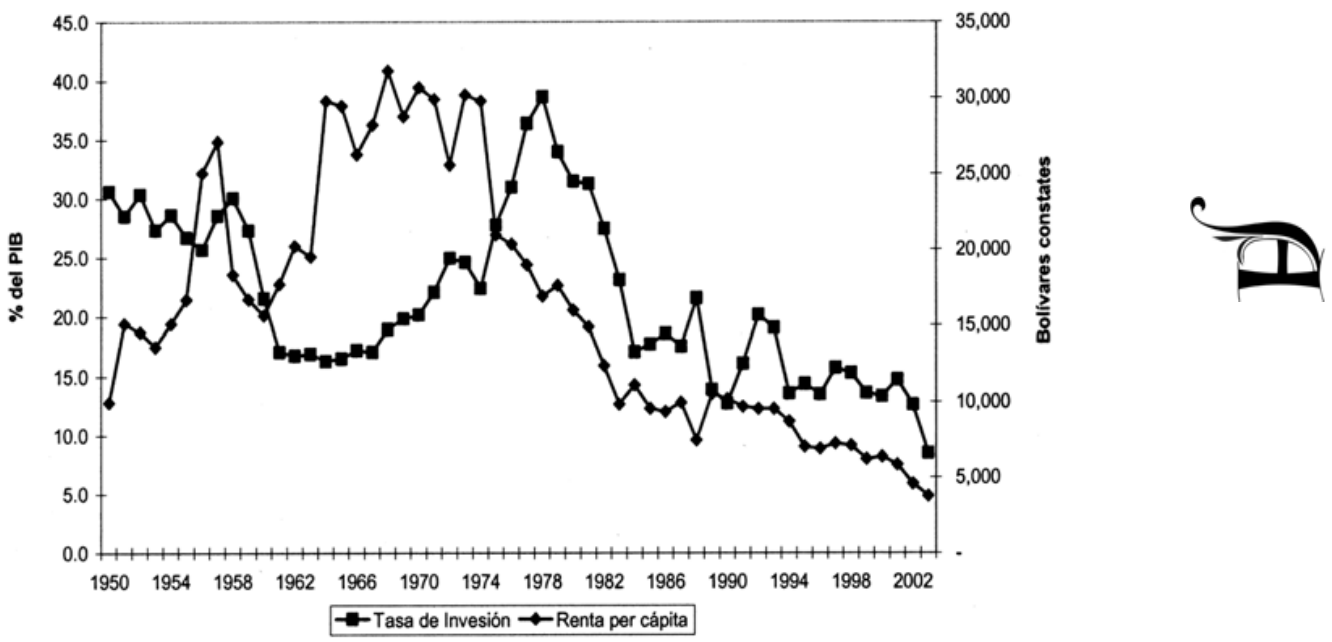

Gráfica 1. Tasa de inversión de capital y renta per capita

Fuente: cálculos propios con base en BCv, Asdrubal Báptista: Bases Cuantitativas de la Economía Venezolana: 1830-1995. Para el periodo 1996-2003.

\section{Renta petrolera y función de crecimiento para Venezuela}

Para establecer la importancia de la renta en la economía venezolana estimaremos el efecto que ésta tiene en el producto de la economía. La función que ha de estimarse se presenta como los logaritmos de las variables en términos per capita. La función del producto per capita queda como sigue:

$$
\begin{gathered}
y_{t}=\alpha_{0}+\alpha_{1} r_{t}+\alpha_{2} s y_{t}+\alpha_{3}(n+\delta)_{t}+\varepsilon_{t} \\
\alpha_{0}, \alpha_{1} \mathrm{y} \alpha_{2}>0 \mathrm{y} \alpha_{3}<0
\end{gathered}
$$

Donde $r$ es la renta per capita, sy es la tasa de inversión en capital y $\left(n++^{\prime}\right)$ es la tasa de crecimiento de la población y de depreciación de los bienes de capital.

El efecto de la renta como fuente de financiamiento de la actividad económica se ha visto disminuido, sobre todo a partir de finales de los setenta, cuando la economía entra en un periodo de fuerte recesión. Dado que se analiza el periodo de 1950 a 2003, se incluirá un conjunto de variables interactivas que nos permitan medir el efecto de la renta, así como su importancia a lo largo de este periodo. Se incluirán igualmente variables de carácter interactivo para la inversión de capital. Así, la expresión (10) se volverá a estimar incorporando variables de carácter interactivo:

$$
\begin{gathered}
y_{t}=\alpha_{0}+\alpha_{1} r_{t} * f_{t}+\alpha_{2} r_{t}^{*} q_{t}+\alpha_{3} s y_{t} * f_{t}+\alpha_{4} s y_{t} * q_{t}+\alpha_{5}(n+\delta)_{t} * f_{t}+\alpha_{6}(n+\delta)_{t} * q_{t}+\varepsilon_{t} \\
\alpha_{0}, \alpha_{1} \mathrm{y} \alpha_{3}>0 \alpha_{2}, \alpha_{4}, \alpha_{5} \mathrm{y} \alpha_{6}<0
\end{gathered}
$$


Donde $f_{t}$ y $q_{t}$ son variables interactivas que intentan recoger el efecto de las variables explicativas antes y después del quiebre estructural observado en la economía venezolana en 1978.

Recordemos que la renta actúa financiando la inversión de capital y el consumo, tanto de origen nacional como importado. De aquí la relevancia de ver su efecto en el producto de la economía en una función que incluye, a su vez, variables de carácter interactivo que permitan medir qué tan estable ha sido el aporte o la importancia de la renta petrolera para motorizar el aparato productivo en el caso venezolano.

Igualmente se llevará a cabo la estimación del producto con la intención de extraer por residuo la productividad total de los factores ( $p t f)$; para ello se partirá de una función sin constante que incluye como variables al factor trabajo junto con la renta y la inversión de capital como principales factores productivos. Dicha función queda de la siguiente forma:

$$
\begin{gathered}
y_{t}=\alpha_{0}+\alpha_{1} r_{t}+\alpha_{2} s y_{t}+\alpha_{3} l_{t}+\varepsilon_{t} \\
\alpha_{1} \text { у } \alpha_{2} \text { y } \alpha_{3}>0
\end{gathered}
$$

Ahora bien, como el parámetro de la productividad total de los factores no es observable directamente, debe obtenerse como residuo a partir de la ecuación (11), una vez conocidos los valores de los parámetros que acompañan a las variables explicativas. En ese sentido, la $p t f$ se obtendrá por medio de la siguiente expresión:

$$
p t f=y_{t}-\alpha_{0}+\alpha_{1} r_{t}+\alpha_{2} s y_{t}+\alpha_{3} l_{t}
$$

Así la productividad total de los factores se calcula como residuo de la estimación de un modelo del producto con respecto de sus principales factores como son, en este caso, la renta, el capital y el trabajo.

Pero antes de llevar a cabo esas estimaciones, se hace necesario realizar algunas otras, además de pruebas previas sobre las series con la intención. Por un lado, hay que completar la serie de la renta petrolera, ya que ésta sólo está disponible hasta 1995; y por otro, llevar a cabo un test de raíces unitarias, ya que las variables por utilizarse para las estimaciones pueden ser series no estacionarias, lo cual se traduciría en correlaciones espurias; es decir, las relaciones en las variables se dan por la marcada tendencia mostrada en las variables más que en la verdadera relación que subyace en las series. ${ }^{5}$

La serie de la renta petrolera está disponible desde 1950 hasta 1995 y se tomó de Baptista (1997). Para completar la serie hasta 2003, se llevará a cabo una estimación a partir del siguiente modelo:

5 En este caso se dice que las variable siguen una caminata aleatoria, su media y su varianza no permanecen constantes a lo largo del tiempo.

\section{DeSarrollo}




$$
\begin{aligned}
& R_{t}=\beta_{0}+\beta_{1} Y_{t}^{*}+\beta_{2} P_{t}^{*}+\beta_{3} T_{t}+\varepsilon_{t} \\
& \quad \varepsilon_{\mathrm{t}} \sim \mathrm{N}\left(0, \sigma^{2}\right)
\end{aligned}
$$

En (13) se parte del supuesto de que la renta petrolera es una función del producto petrolero $\left(Y_{t}^{*}\right)$, de los precios del petróleo $\left(P_{t}^{*}\right)$, de un componente tendencial $\left(T_{t}\right)$ y de un término de error aleatorio $\varepsilon_{t}$, el cual sigue una distribución normal. ${ }^{6}$ De esta función todas las variables resultaron significativas estadísticamente a $1 \%$ y con el signo esperado.

La serie de la renta petrolera para el periodo 1996-2003 se completa con la renta estimada a partir de la ecuación (13). Una vez hecho esto, se incluirá como variable explicativa en el modelo de crecimiento expresado en (10), junto a las variables interactivas, para medir su efecto en esta variable antes y después del quiebre de la actividad económica.

Por otro lado, para comprobar si las series por ser utilizadas en los modelos presentan raíces unitarias, o sea, si son no estacionarias, se aplicó en éstas la prueba de Dickey-Fuller aumentado; de aquí se desprende que todas las variables son integradas de orden uno, es decir, presentan una raíz unitaria, no son estacionarias en niveles sino en primeras diferencias. En el Cuadro 1 se pueden observar los resultados obtenidos de estas pruebas. ${ }^{7}$

Cuadro 1

Test de Dickey-Fuller Aumentado

\begin{tabular}{cccc}
\hline Variable & Valor en niveles & Valor en primera diferencia & Valor crítico a 1\% \\
\hline$y_{t}$ & -1.11 & -4.77 & -4.14 \\
$r_{t}$ & -3.06 & -5.12 & -4.14 \\
$s y_{t}$ & -1.19 & -4.77 & -4.14 \\
$n+d$ & -2.81 & 4.94 & -4.14 \\
\hline
\end{tabular}

Una vez identificado el orden de integración de las variables, se procede a llevar a cabo la estimación de la ecuación de crecimiento expresada en (10) en primeras diferencias de sus logaritmos, con la intención de medir el efecto de las variables renta per capita, de la tasa de inversión de capital y de la tasa de crecimiento de la población en el crecimiento del ingreso per capita. ${ }^{8}$

En el Cuadro 2 del anexo se pueden observar los resultados de la estimación. El modelo en su conjunto es significativo a un nivel de confianza de $99 \%$. Por otro lado, la capacidad explicativa del modelo es bastante buena, pues alcanzó 86\%. Tal como se esperaba, la renta petrolera y la inversión de capital por trabajador tienen un efecto positivo en el

6 Se incluyó un término autorregresivo de orden uno AR(1) con la intención de corregir el modelo ante la presencia de autocorrelación.

7 Estos resultados fueron corroborados mediante la aplicación de la prueba de Phillips $\sim$ Perron.

8 Se incluyó, además, un conjunto de variables de comportamiento para recoger puntos de quiebres en las relaciones de las variables. 
Cuadro 2*

Variable Dependiente: $\mathrm{D}\left(y_{t}\right)$

Periodo: 19512003

Observaciones: 53

\begin{tabular}{lcc}
\hline \multicolumn{1}{c}{ Variable } & Coeficiente & Estadístico $-t$ \\
\hline$C$ & 0.010 & $2.119^{\mathrm{a}}$ \\
$D\left(r_{t}\right)$ & 0.293 & $9.357^{\mathrm{a}}$ \\
$D\left(s y_{t}\right)$ & 0.179 & $7.282^{\mathrm{a}}$ \\
$D(n+d)$ & -0.030 & $-3.974^{\mathrm{a}}$ \\
$F_{84}$ & -0.038 & $-3.525^{\mathrm{a}}$ \\
$F_{89}$ & -0.110 & $-7.544^{\mathrm{a}}$ \\
$F_{96}$ & -0.0314 & $-3.430^{\mathrm{a}}$ \\
$F_{02}$ & -0.0316 & $-3.045^{\mathrm{a}}$ \\
$\mathrm{R}^{2}$ & 0.860 & \\
F-statistic $^{\text {Durbin-Watson }}$ & 39.794 & \\
\hline
\end{tabular}

Nota: a el coeficiente es significativo al $1 \%$; ${ }^{\mathrm{b}}$ significativo al $5 \%$; ${ }^{\mathrm{c}}$ no es significativo.

* No se encontró evidencia que permitiera rechazar la hipótesis de normalidad, no autocorrelación y homocedasticidad de los residuos.

Cuadro 3*

Variable Dependiente: $\mathrm{D}\left(y_{t}\right)$

Periodo: 1952- 2003

Observaciones 52

\begin{tabular}{lcc}
\hline Variable & Coeficiente & Estadistico $-t$ \\
\hline $\mathrm{C}$ & 0.009 & $1.808^{\mathrm{c}}$ \\
$D\left(r_{t}\right) * f_{t}$ & 0.309 & $9.064^{\mathrm{a}}$ \\
$D\left(r_{t}\right) * q_{t}$ & 0.255 & $6.340^{\mathrm{a}}$ \\
$D\left(s y_{t}\right) * f_{t}$ & 0.193 & $4.198^{\mathrm{a}}$ \\
$D\left(s y_{t} *^{*} q_{t}\right.$ & 0.180 & $6.412^{\mathrm{a}}$ \\
$D(n+d) * f_{t}$ & 0.243 & $1.264^{\mathrm{c}}$ \\
$D(n+d) * q_{t}$ & -0.031 & $-4.901^{\mathrm{a}}$ \\
$F_{84}$ & -0.038 & $-3.490^{\mathrm{a}}$ \\
$F_{89}$ & -0.095 & $-5.281^{\mathrm{a}}$ \\
$F_{96}$ & -0.032 & $-3.455^{\mathrm{a}}$ \\
$F_{02}$ & -0.039 & $-3.718^{\mathrm{a}}$ \\
$\mathrm{R}^{2}$ & 0.867 & \\
F-statistic & 27.547 & \\
Durbin-Watson & 1.426 & \\
\hline
\end{tabular}

Nota: ${ }^{\mathrm{a}}$ el coeficiente es significativo al $1 \%$; ${ }^{\mathrm{b}}$ significativo al $5 \% ;^{\mathrm{c}}$ no es significativo.

* No se encontró evidencia que permitiera rechazar la hipótesis de normalidad, no autocorrelación y homocedasticidad de los residuos.

\section{Desarrollo}


crecimiento a lo largo del periodo de análisis. ${ }^{9}$ Igualmente la variable crecimiento de la población resultó significativa y con signo negativo. Tal como lo establece la teoría económica, un mayor crecimiento de la población traerá consigo un menor producto per capita si el stock de capital permanece constante o, bien, disminuye, como ha sido el caso en Venezuela a partir de finales de la década de los setenta.

En el Cuadro 3 se puede apreciar el modelo para el crecimiento con la inclusión de un conjunto de variables de carácter interactivo (ecuación 10.1). El modelo sigue siendo significativo a un nivel de confianza de $99 \%$, con una capacidad explicativa de $86.7 \%$ de acuerdo con $R^{2}$.

Como se observa, la renta ha experimentado una disminución en su impacto sobre el crecimiento; su efecto es menor a partir de 1978 en comparación con el periodo que va de 1950 a 1977, cuando la renta tuvo su mayor aporte a la actividad económica. El descenso del crecimiento económico en el caso venezolano se explica, en parte, por la caída en la renta percibida por el Estado. Al verse éste con recursos insuficientes no puede continuar llevando a cabo su papel de benefactor y promotor principal de la actividad económica.

A partir de entonces, el Estado usó la devaluación del signo monetario y el endeudamiento como sus principales políticas para hacerse de recursos que le permitieran seguir financiando su política expansiva de la actividad económica. Esto trajo consigo severos desequilibrios en las cuentas macroeconómicas, que se reflejan en elevados niveles de liquidez, altas y volátiles tasas de inflación y de interés.

Al igual que en el caso de la renta, se aprecia una disminución considerable en el parámetro de la inversión de capital en la economía; la inversión bruta de capital fijo por trabajador tiene un efecto menor sobre el crecimiento: se está invirtiendo menos en la economía después de 1978; incluso la inversión no alcanza ni siquiera para reponer los bienes de capital que han llegado al final de su vida útil.

En la Gráfica 2 se puede apreciar tanto el PIB per capita como el stock de capital por trabajador ocupado en la economía. Claramente se observa cómo a partir de 1978 se produce una caída severa y continúa en la acumulación de capital por trabajador, a la par de un descenso en el PIB per capita.

En el caso de la tasa de crecimiento de la población, llama la atención cómo la variable no es significativa para el periodo 1950-1977. Este comportamiento puede ser explicado precisamente porque es en este lapso cuando la renta hace su mayor contribución a la expansión del aparato productivo; la inversión de capital tanto pública como privada estaban creciendo a tasa superiores a la de la población, con lo cual se contrarrestaba su efecto. Ahora, a partir de la caída de la renta y con ello de la inversión de capital, el efecto del

9 Las variables de comportamiento incluidas resultaron significativas estadísticamente con signo negativo en todos los casos, y expresan caídas apreciables en el ingreso per capita en cada uno de esos momentos, lo que resalta la caída observada a partir de 1989. 


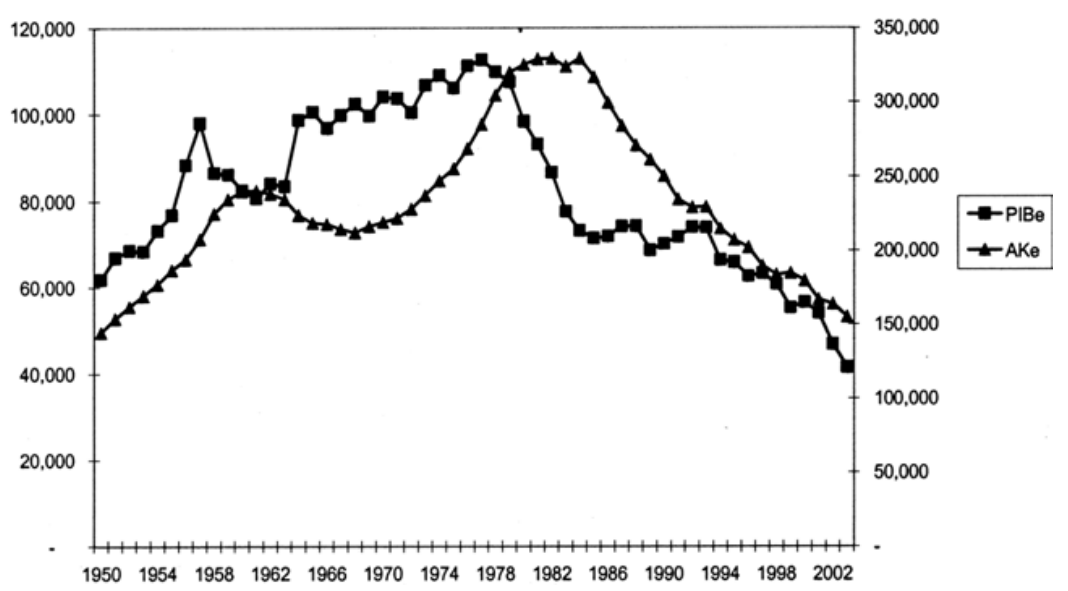

Gráfica 2. PIB per capita y stock de capital por trabajador ocupado expresado en bolívares constantes. Fuente: cálculos propios con base en BCV.

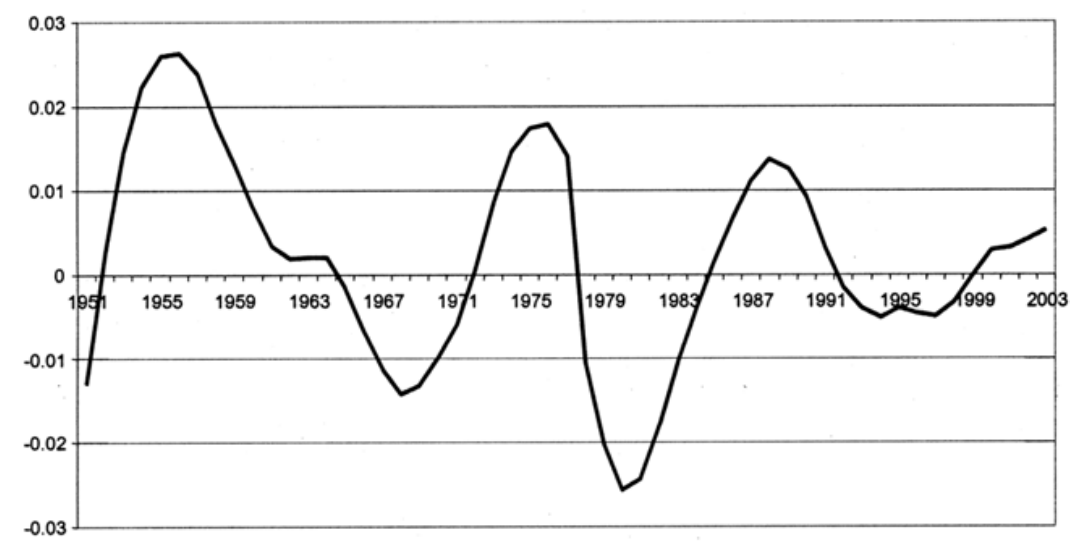

Gráfica 3. Productividad total de los factores expresado en logaritmos y suavizado mediante el filtro de Hodrick-Prescot. Cálculos propios.

crecimiento de la población en la actividad económica ha sido negativa, tal como se observa en el coeficiente de la variable para el periodo de finales de los setenta. La inversión ha sido insuficiente para compensar, por un lado, el crecimiento de la población y la depreciación de los bienes de capital, por otro, lo cual se ha traducido en una disminución del PIB per capita.

En la Gráfica 3 se puede apreciar el comportamiento cíclico de la productividad total de los factores obtenido de la estimación de las funciones 11 sin constante y de la función 12. Como se observa, la productividad total de los factores ha venido descendiendo de manera sostenida a lo largo del periodo de análisis. Salvo algunos repuntes en los años ochenta y noventa, la productividad de los factores no ha alcanzado los niveles observados

\section{DeSarrollo}


Cuadro 4*

Variable Dependiente: $\mathbf{D}\left(\mathbf{y}_{\mathbf{t}}\right)$

Periodo: 19512003

Observaciones: 53

\begin{tabular}{lcc}
\hline \multicolumn{1}{c}{ Variable } & Coeficiente & Estadístico $-t$ \\
\hline$C$ & 0.007 & $2.888^{\mathrm{a}}$ \\
$D\left(r_{t}\right) * f_{t}$ & 0.314 & $11.046^{\mathrm{a}}$ \\
$D\left(r_{t}\right) * q_{t}$ & 0.231 & $7.404^{\mathrm{a}}$ \\
$D\left(s_{t}\right) * f_{t}$ & 0.204 & $7.460^{\mathrm{a}}$ \\
$D\left(s_{t}\right) * q_{t}$ & 0.175 & $6.649^{\mathrm{a}}$ \\
$p t f_{t} f_{t}$ & 1.304 & $5.017^{\mathrm{a}}$ \\
$p t f * q_{t}$ & 1.162 & $6.354^{\mathrm{a}}$ \\
$\mathrm{R}^{2}$ & 0.925 & \\
Estadístico -F & 51.873 & \\
Durbin-Watson & 2.646 & \\
\hline
\end{tabular}

Nota: ${ }^{a}$ el coeficiente es significativo al $1 \%$; ${ }^{\mathrm{b}}$ significativo al $5 \% ;{ }^{\mathrm{c}}$ no es significativo

* De acuerdo con las pruebas realizadas, no se pudo rechazar la hipótesis de no violación de los supuestos clásicos del modelo de regresión lineal de no autocorrelación, normalidad y homocedasticidad de los residuos.

en los cincuenta, sesenta y parte de los setenta. Lo descendente se corresponde con un menor nivel de capital por trabajador y un incremento en la capacidad ociosa, lo cual se traduce en uso ineficiente de los factores productivos. De igual manera, la caída de ptf coincide con una disminución en la renta per capita en la economía venezolana.

Al incluir la $p t f$ en nuestro modelo obtenemos como principal resultado que la productividad de los factores se ha visto reducida a partir de finales de los setenta, tal como se observa en el Cuadro 4. Su efecto en el crecimiento es menor, pero es la variable de mayor importancia para explicar el crecimiento; es decir, éste está asociado no sólo a un mayor stock de capital por trabajador acumulado y a una renta per capita creciente o al mejoramiento del factor humano, sino a su productividad, la cual tiene mucho que ver con el progreso tecnológico, el grado de especialización y aprovechamiento de manera eficiente de esos factores productivos.

La variable renta observa menor contribución a la generación del crecimiento a partir de la variable interactiva de finales de los setenta, pero ésta sigue teniendo una importancia en el crecimiento incluso superior a la de la inversión de capital. Ahora bien, es osado concluir que hay un aparente agotamiento del modelo rentístico que basaba el crecimiento en una renta creciente. No obstante, éste parece ser el caso a la luz de los resultados obtenidos. En palabras de Baptista (2004:84), "el colapso del rentismo ni prefigura ni saluda una etapa posterior. Meramente, con su final histórico, sólo se da una apertura de posibilidades, toda vez que ha quedado despejado el camino al que la renta, en los momentos finales del proceso que ella signa de modo determinante, terminó por entrabar y obstaculizar". 
Según Mommer (1995), el colapso del capitalismo rentístico es un hecho en la economía venezolana; no así la renta petrolera, la cual, si bien ha bajado, sigue teniendo un alto peso para hacerle frente a los compromisos de la deuda externa. Por otro lado, su relación con el capital invertido sigue siendo muy alta. Según el autor, apenas se ha iniciado la reorganización del sector petrolero para poner fin a su aislamiento estructural en la economía internacional y dentro de la economía nacional.

Igual comportamiento se aprecia en el caso de la inversión de capital, la cual ve reducida su importancia como estimuladora del crecimiento; los niveles menores de inversión observados en la economía a partir de finales de los setenta han tenido un efecto adverso en el crecimiento de la producción en el país.

La inversión de capital y la productividad total de los factores constituyen las claves del crecimiento. La acumulación de capital productivo bien aprovechado, que propicie la especialización, el progreso tecnológico, el crecimiento del capital humano, la expansión de las fronteras productivas más allá de la actividad petrolera, parece ser la opción al modelo rentístico que prevalece en la economía venezolana.

\section{Conclusiones}

A la luz de los resultados se hace evidente el debilitamiento del modelo rentista predominante en la economía venezolana hasta la actualidad. La renta petrolera no ha tenido el mismo efecto en la economía después de que se alcanzó el máximo crecimiento en 1977, para luego entrar en un periodo de recesión económica que aún no se ha detenido. Ahora bien, de acuerdo con los resultados obtenidos, la renta sigue teniendo una importancia significativa en el crecimiento, pues el uso de este recurso estimula el crecimiento de manera directa mediante el gasto del Estado, e indirecta por medio de las múltiples externalidades positivas que este recurso genera en el resto de los sectores productivos de la economía.

El descenso de la actividad económica coincide con dos aspectos importantes: a) la caída en la inversión de capital productivo, sobre todo del sector privado, originada por la incapacidad de la demanda para absorber la expansión de la capacidad productiva, y $b$ ) la caída paralela en la renta petrolera percibida por la nación a partir de entonces. La renta fue la principal fuente de financiamiento de la actividad económica y, en cierta forma, también del sobrecalentamiento de la misma. Es decir, dentro de las razones causales para explicar el proceso de desacumulación de capital se encuentra la disminución de la renta, la cual hizo imposible sostener la tasa de inversión que permitiera mantener la relación del estado estacionario; la tasa de inversión no compensa la depreciación del stock de capital ni la tasa de crecimiento de la población.

La caída simultanea en la renta per capita y del stock de capital por trabajador ocupado se ha visto traducido en una disminución de la productividad de los factores tal como se desprende de los resultados. Ha habido un incremento de la capacidad ociosa y un

\section{DeSarrollo}


desaprovechamiento de los recursos; los factores productivos no están siendo utilizados de manera eficiente. Por otro lado, la $p t f$ es la variable de mayor importancia para explicar el crecimiento, lo que hace necesario enfocar los esfuerzos en llevar a cabo mayores inversiones en nuevas tecnologías, ampliar la capacidad productiva mediante el uso eficiente de los factores productivos existentes, lograr mayor inversión en capital humano, así como promover menor dependencia del gasto del Estado.

\section{Bibliografía}

Auty, R. M., Resource Based Industrializatión: Sowing The Oil in Eight Developing Countries, Oxford University Press, Oxford, 1990.

Baptista, Asdrúbal y Bernardo Mommer, El Ingreso Petrolero y la Capacidad de absorción de Capital de la Economía Venezolana, Caracas, IESA, 1985.

Baptista, Asdrúbal, Bases Cuantitativas de la Economía Venezolana 1830-1995, Caracas, Fundación Polar, 1997, pp. 337.

_ El Relevo del Capitalismo Rentístico: Hacia un Nuevo Balance de Poder, Caracas, Fundación Polar, 2004.

Corden, W. Max, "The Economic Effects of a Boooming Sector", en Internacional Social Sciener Journal, núm. 35, Oxford, 1983.

"Booming Sector and Dutch Disease Economics: Survey and Consolidation", en Oxford Economics Paper, vol. 40, núm. 35, 1984.

Galindo, Miguel y Graciela Malgasini, Crecimiento Económico Principales. Teorías desde Keynes, McGraw-Hill, España, 1994, pp. 145.

Gimeno González, Juan y Jesús Ruiz Concepción, Introducción a la Macroeconomía, Editorial Mc Graw- Hill, España, 2000, pp. 417.
Harrod, Roy, Dinámica económica, Madrid, Alianza Editorial, 1979, pp 325.

Kalecki, Michal Teoría de la Dinámica Económica, FCE, México, 1997, pp. 628.

López, Odalis, "La Enfermedad Holandesa y la Economía Venezolana en el Período 1973-1982 y el Colapso del Capitalismo Rentístico", en Revista Venezolana. de Economía y Ciencias Sociales, vol. 7 núm. 2, mayo-agosto, 2001, pp. 67-107.

Mommer, B., Análisis estructural de una economía petrolera: Venezuela, Caracas, Pdvsa, 1995.

Rodríguez, F. y J. D. Sachs, "Why do resource abundant economies grow more slowly? A new explanation and an application to Venezuela", en Journal of Economic Growth, 1999, pp. 277-303.

Sachs, Jeffrey y Warner, Andrew, Natural Resource Abundance and Economic Growth, National Bureau of Economic Research, Working Paper 5398, 1995, pp. 47.

Schiesser, Reinier y Silva José, La renta Petrolera y El Crecimiento Económico de Venezuela: Análisis del Periodo 1913-1995, Banco Central de Venezuela, Series documentos de Trabajo, 2000, pp. 44.

\section{DeSarrrollo}

